\title{
Article
}

\section{On Hermite-Hadamard-Fejér type integral inequalities for generalized convex functions via local fractional integrals}

\author{
Mehmet Zeki Sarikaya ${ }^{1}$ and Necmettin Alp ${ }^{1, *}$ \\ 1 Department of Mathematics, Faculty of Science and Arts, Düzce University, Düzce, Turkey.; \\ sarikayamz@gmail.com(M.Z.S) \\ * Correspondence: placenn@gmail.com
}

Received: 14 May 2019; Accepted: 26 August 2019; Published: 9 September 2019.

Abstract: In this paper, we extend some estimates of the right hand side of a Hermite- Hadamard-Fejér type inequality for generalized convex functions whose derivatives absolute values are generalized convex via local fractional integrals.

Keywords: Hermite-Hadamard's inequality, generalized convex function, local fractional integral, generalized Hölder's inequality.

MSC: 26A33, 26D07, 26D10.

\section{Introduction}

T he definition of convex function is known in the literature:

Definition 1 (Convex function). The function $f:[a, b] \subset R \rightarrow R$, is said to be convex if the following inequality holds

$$
f(t x+(1-t) y) \leq t f(x)+(1-t) f(y)
$$

for all $x, y \in[a, b]$ and $t \in[0,1]$. We say that $f$ is concave if $(-f)$ is convex.

The classical Hermite-Hadamard inequality (1) which was first published in [1] gives us an estimate of the mean value of a convex function $f: I \rightarrow \mathbb{R}$.

$$
f\left(\frac{a+b}{2}\right) \leq \frac{1}{b-a} \int_{a}^{b} f(x) d x \leq \frac{f(a)+f(b)}{2}
$$

In [2], Bullen proved the following inequality (1), which is known as Bullen's inequality for convex function.

Let $f: I \subset \mathbb{R} \rightarrow \mathbb{R}$ be a convex function on the interval $I$ of real numbers and $a, b \in I$ with $a<b$. The inequality

$$
\frac{1}{b-a} \int_{a}^{b} f(x) d x \leq \frac{1}{2}\left[f\left(\frac{a+b}{2}\right)+\frac{f(a)+f(b)}{2}\right] .
$$

The history of Bullen's inequality can be found in [3]. Surveys on various generalizations and developments in inequality theory can be found in [4] and [5]. Recently in [6], the authors established Bullen's inequality for twice differentiable functions. In the case where $f$ is convex then there exists an estimation better than (1).

In [7], Farissi gave the refinement of the inequality (1) as follows:

Theorem 2. Assume that $f: I \rightarrow \mathbb{R}$ is a convex function on $I$. Then for all $\lambda \in[0,1]$, we have

$$
f\left(\frac{a+b}{2}\right) \leq l(\lambda) \leq \frac{1}{b-a} \int_{a}^{b} f(x) d x \leq L(\lambda) \leq \frac{f(a)+f(b)}{2},
$$

where

$$
l(\lambda):=\lambda f\left(\frac{\lambda b+(2-\lambda) a}{2}\right)+(1-\lambda) f\left(\frac{(1+\lambda) b+(1-\lambda) a}{2}\right)
$$


and

$$
L(\lambda):=\frac{1}{2}(f(\lambda b+(1-\lambda) a)+\lambda f(a)+(1-\lambda) f(b)) .
$$

For more information about recent developments in above inequalities, we recommend [5-12].

\section{Preliminaries}

For $0<\alpha \leq 1$, we have the following $\alpha$-type set:

The $\alpha$-type set of integer is defined as: the set $Z^{\alpha}:=\left\{0^{\alpha}, \pm 1^{\alpha}, \pm 2^{\alpha}, \ldots, \pm n^{\alpha}, \ldots\right\}$.

The $\alpha$-type set of the rational numbers is defined as: $Q^{\alpha}:=\left\{m^{\alpha}=\left(\frac{p}{q}\right)^{\alpha}: p, q \in Z, q \neq 0\right\}$.

The $\alpha$-type set of the irrational numbers is defined as: $J^{\alpha}:=\left\{m^{\alpha} \neq\left(\frac{p}{q}\right)^{\alpha}: p, q \in Z, q \neq 0\right\}$.

The $\alpha$-type set of the real line numbers is defined as: $R^{\alpha}:=Q^{\alpha} \cup J^{\alpha}$.

If $a^{\alpha}, b^{\alpha}$ and $c^{\alpha}$ belongs the set $R^{\alpha}$ of real line numbers, then we have

1. $a^{\alpha}+b^{\alpha}$ and $a^{\alpha} b^{\alpha}$ belongs the set $R^{\alpha}$;

2. $a^{\alpha}+b^{\alpha}=b^{\alpha}+a^{\alpha}=(a+b)^{\alpha}=(b+a)^{\alpha}$;

3. $a^{\alpha}+\left(b^{\alpha}+c^{\alpha}\right)=(a+b)^{\alpha}+c^{\alpha}$;

4. $a^{\alpha} b^{\alpha}=b^{\alpha} a^{\alpha}=(a b)^{\alpha}=(b a)^{\alpha}$;

5. $a^{\alpha}\left(b^{\alpha} c^{\alpha}\right)=\left(a^{\alpha} b^{\alpha}\right) c^{\alpha}$;

6. $a^{\alpha}\left(b^{\alpha}+c^{\alpha}\right)=a^{\alpha} b^{\alpha}+a^{\alpha} c^{\alpha}$;

7. $a^{\alpha}+0^{\alpha}=0^{\alpha}+a^{\alpha}=a^{\alpha}$ and $a^{\alpha} 1^{\alpha}=1^{\alpha} a^{\alpha}=a^{\alpha}$.

Gao-Yang-Kang's $[13,14]$ introduced the idea the local fractional derivative and local fractional integral.

Definition 3. [13] A non-differentiable function $f: R \rightarrow R^{\alpha}, x \rightarrow f(x)$ is local fractional continuous at $x_{0}$, if for any $\varepsilon>0$, there exists $\delta>0$, such that

$$
\left|f(x)-f\left(x_{0}\right)\right|<\varepsilon^{\alpha}
$$

holds for $\left|x-x_{0}\right|<\delta$, where $\varepsilon, \delta \in R$. We denote the set of all locally fractional continuous functions on $(a, b)$ by $C_{\alpha}(a, b)$.

Definition 4. [13] The local fractional derivative of $f(x)$ of order $\alpha$ at $x=x_{0}$ is defined as:

$$
f^{(\alpha)}\left(x_{0}\right)=\left.\frac{d^{\alpha} f(x)}{d x^{\alpha}}\right|_{x=x_{0}}=\lim _{x \rightarrow x_{0}} \frac{\Delta^{\alpha}\left(f(x)-f\left(x_{0}\right)\right)}{\left(x-x_{0}\right)^{\alpha}},
$$

where $\Delta^{\alpha}\left(f(x)-f\left(x_{0}\right)\right) \cong \Gamma(\alpha+1)\left(f(x)-f\left(x_{0}\right)\right)$.

If there exists $f^{(k+1) \alpha}(x)=\overbrace{D_{x}^{\alpha} \ldots D_{x}^{\alpha}}^{k+1 \text { times }} f(x)$ for any $x \in I \subseteq R$, then we say that $f \in D_{(k+1) \alpha}(I)$, where $k=0,1,2, \ldots$

Definition 5. [13] Let $f(x) \in C_{\alpha}[a, b]$. Then the local fractional integral is defined by,

$$
{ }_{a} I_{b}^{\alpha} f(x)=\frac{1}{\Gamma(\alpha+1)} \int_{a}^{b} f(t)(d t)^{\alpha}=\frac{1}{\Gamma(\alpha+1)} \lim _{\Delta t \rightarrow 0} \sum_{j=0}^{N-1} f\left(t_{j}\right)\left(\Delta t_{j}\right)^{\alpha},
$$

with $\Delta t_{j}=t_{j+1}-t_{j}$ and $\Delta t=\max \left\{\Delta t_{1}, \Delta t_{2}, \ldots, \Delta t_{N-1}\right\},\left[t_{j}, t_{j+1}\right], j=0, \ldots, N-1$ and $a=t_{0}<t_{1}<\ldots<$ $t_{N-1}<t_{N}=b$ is partition of interval $[a, b]$.

Here, it follows that ${ }_{a} I_{b}^{\alpha} f(x)=0$ if $a=b$ and ${ }_{a} I_{b}^{\alpha} f(x)={ }_{b} I_{a}^{\alpha} f(x)$ if $a<b$. If for any $x \in[a, b]$, there exists ${ }_{a} I_{x}^{\alpha} f(x)$, then we say that $f(x) \in I_{x}^{\alpha}[a, b]$.

Definition 6 (Generalized convex function). [13] Let $f: I \subseteq R \rightarrow R^{\alpha}$. For any $x_{1}, x_{2} \in I$ and $\lambda \in[0,1]$, if

$$
f\left(\lambda x_{1}+(1-\lambda) x_{2}\right) \leq \lambda^{\alpha} f\left(x_{1}\right)+(1-\lambda)^{\alpha} f\left(x_{2}\right)
$$

holds, then $f$ is a generalized convex function on $I$. 
Here are two basic examples of generalized convex functions:

1. $f(x)=x^{\alpha p}, x \geq 0, p>1$;

2. $f(x)=E_{\alpha}\left(x^{\alpha}\right), x \in R$ where $E_{\alpha}\left(x^{\alpha}\right)=\sum_{k=0}^{\infty} \frac{x^{\alpha k}}{\Gamma(1+k \alpha)}$ is the Mittag-Leffler function.

Theorem 7. [15] Let $f \in D_{\alpha}(I)$, then the followings are equivalent

a) $f$ is a generalized convex function on $I$.

b) $f^{(\alpha)}$ is an increasing function on $I$.

c) for any $x_{1}, x_{2} \in I$,

$$
f\left(x_{2}\right)-f\left(x_{1}\right) \geq \frac{f^{(\alpha)}\left(x_{1}\right)}{\Gamma(1+\alpha)}\left(x_{2}-x_{1}\right)^{\alpha} .
$$

Corollary 8. [15] Let $f \in D_{2 \alpha}(a, b)$. Then $f$ is a generalized convex function (or a generalized concave function) if and only if

$$
f^{(2 \alpha)}(x) \geq 0\left(\text { or } f^{(2 \alpha)}(x) \leq 0\right)
$$

for all $x \in(a, b)$.

Lemma 9. [13]

1. (Local fractional integration is anti-differentiation) Suppose that $f(x)=g^{(\alpha)}(x) \in C_{\alpha}[a, b]$, then we have

$$
{ }_{a} I_{b}^{\alpha} f(x)=g(b)-g(a)
$$

2. (Local fractional integration by parts) Suppose that $f(x), g(x) \in D_{\alpha}[a, b]$ and $f^{(\alpha)}(x), g^{(\alpha)}(x) \in C_{\alpha}[a, b]$, then we have

$$
{ }_{a} I_{b}^{\alpha} f(x) g^{(\alpha)}(x)=\left.f(x) g(x)\right|_{a} ^{b}-{ }_{a} I_{b}^{\alpha} f^{(\alpha)}(x) g(x)
$$

Lemma 10. [13] We have

1. $\frac{d^{\alpha} x^{k \alpha}}{d x^{\alpha}}=\frac{\Gamma(1+k \alpha)}{\Gamma(1+(k-1) \alpha)} x^{(k-1) \alpha}$

2. $\frac{1}{\Gamma(\alpha+1)} \int_{a}^{b} x^{k \alpha}(d x)^{\alpha}=\frac{\Gamma(1+k \alpha)}{\Gamma(1+(k+1) \alpha)}\left(b^{(k+1) \alpha}-a^{(k+1) \alpha}\right), k \in R$.

Lemma 11. [13] Suppose that $f(x) \in C_{\alpha}[a, b]$, then

$$
\frac{d^{\alpha}\left({ }_{a} I_{x}^{\alpha} f(t)\right)}{d x^{\alpha}}=f(x) a<x<b
$$

Lemma 12 (Generalized Hölder's inequality). [13] Let $f, g \in C_{\alpha}[a, b], p, q>1$ with $\frac{1}{p}+\frac{1}{q}=1$, then

$$
\frac{1}{\Gamma(\alpha+1)} \int_{a}^{b}|f(x) g(x)|(d x)^{\alpha} \leq\left(\frac{1}{\Gamma(\alpha+1)} \int_{a}^{b}|f(x)|^{p}(d x)^{\alpha}\right)^{\frac{1}{p}}\left(\frac{1}{\Gamma(\alpha+1)} \int_{a}^{b}|g(x)|^{q}(d x)^{\alpha}\right)^{\frac{1}{q}} .
$$

In [15], Mo et al. proved the following generalized Hermite-Hadamard inequality for generalized convex function:

Theorem 13 (Generalized Hermite-Hadamard inequality). Let $f(x) \in I_{x}^{(\alpha)}[a, b]$ be a generalized convex function on $[a, b]$ with $a<b$. Then

$$
f\left(\frac{a+b}{2}\right) \leq \frac{\Gamma(1+\alpha)}{(b-a)^{\alpha}} a_{b}^{\alpha} f(x) \leq \frac{f(a)+f(b)}{2^{\alpha}} .
$$

Sarikaya and Budak [16] gave a weighted generalization of the inequality (2). 
Theorem 14 (Hermite-Hadamard-Fejér inequality). Let $f(x) \in I_{x}^{(\alpha)}[a, b]$ be a generalized convex function on $[a, b]$ with $a<b$. If $g:[a, b] \rightarrow R^{\alpha}$ is nonnegative, local fractional integrable and symmetric $\frac{a+b}{2}$, then the following inequality for local fractional integrals hold

$$
f\left(\frac{a+b}{2}\right){ }_{a} I_{b}^{(\alpha)} g(x) \leq{ }_{a} I_{b}^{(\alpha)} f(x) g(x) \leq \frac{f(a)+f(b)}{2^{\alpha}}{ }_{a} I_{b}^{(\alpha)} g(x) .
$$

The interested reader can read [12-21].

In this paper, we firstly establish generalized Hermite-Hadamard-Fejér inequality via local fractional integrals. Then, we obtain several inequalities related the right side of this inequality using the local fractional integrals and generalized convex functions.

\section{Main results}

Lemma 15. Let $f(x) \in D_{\alpha}[a, b]$ with $a<b$ and $g(x) \in C_{\alpha}[a, b]$. If $f^{(\alpha)} \in I_{x}^{(\alpha)}[a, b]$, then, for all $x \in[a, b]$, the following identity holds:

$$
f(a)_{a} I_{x}^{(\alpha)} g(s)+f(b)_{x} I_{b}^{(\alpha)} g(s)-{ }_{a} I_{b}^{(\alpha)} f(s) g(s)=\frac{1}{\Gamma(1+\alpha)} \int_{a}^{b}\left[\frac{1}{\Gamma(1+\alpha)} \int_{x}^{t} g(s)(d s)^{\alpha}\right] f^{(\alpha)}(t)(d t)^{\alpha} .
$$

Proof. From local fractional integration by parts, we have

$$
\begin{aligned}
& \frac{1}{\Gamma(1+\alpha)} \int_{a}^{b}\left[\frac{1}{\Gamma(1+\alpha)} \int_{x}^{t} g(s)(d s)^{\alpha}\right] f^{(\alpha)}(t)(d t)^{\alpha} \\
= & {\left[\left(\frac{1}{\Gamma(1+\alpha)} \int_{x}^{t} g(s)(d s)^{\alpha}\right) f(t)\right]_{a}^{b}-\frac{1}{\Gamma(1+\alpha)} \int_{a}^{b} f(t) g(t)(d t)^{\alpha} } \\
= & \frac{1}{\Gamma(1+\alpha)}\left[f(b) \int_{x}^{b} g(s)(d s)^{\alpha}-f(a) \int_{x}^{a} g(s)(d s)^{\alpha}-\int_{a}^{b} f(t) g(t)(d t)^{\alpha}\right] \\
= & \frac{1}{\Gamma(1+\alpha)}\left[f(a) \int_{a}^{x} g(s)(d s)^{\alpha}+f(b) \int_{x}^{b} g(s)(d s)^{\alpha}-\int_{a}^{b} f(t) g(t)(d t)^{\alpha}\right] \\
= & f(a)_{a} I_{x}^{(\alpha)} g(s)+f(b)_{x} I_{b}^{(\alpha)} g(s)-{ }_{a} I_{b}^{(\alpha)} f(s) g(s) .
\end{aligned}
$$

This completes the proof.

Remark 1. In Lemma 15, let $g$ be a symmetric to $(a+b) / 2$ and $x=(a+b) / 2$. Then (4) can be written as

$$
\frac{f(a)+f(b)}{2^{\alpha}}{ }_{a} I_{b}^{(\alpha)} g(s)-{ }_{a} I_{b}^{(\alpha)} f(s) g(s)=\frac{1}{\Gamma(1+\alpha)} \int_{a}^{b}\left[\frac{1}{\Gamma(1+\alpha)} \int_{\frac{a+b}{2}}^{t} g(s)(d s)^{\alpha}\right] f^{(\alpha)}(t)(d t)^{\alpha} .
$$

Theorem 16. Let $f(x) \in D_{\alpha}[a, b]$ with $a<b$ and let $f^{(\alpha)} \in I_{x}^{(\alpha)}[a, b], g(x) \in C_{\alpha}[a, b]$ and $\left|f^{(\alpha)}\right|$ is generalized convex on $[a, b]$. Then, for all $x \in[a, b]$, we have

$$
\begin{aligned}
& \left|f(a)_{a} I_{x}^{(\alpha)} g(s)+f(b)_{x} I_{b}^{(\alpha)} g(s)-{ }_{a} I_{b}^{(\alpha)} f(s) g(s)\right| \\
& \leq\left[\left(\frac{\Gamma(1+\alpha)}{\Gamma(1+2 \alpha)}(b-x)^{\alpha}+\frac{\Gamma(1+2 \alpha)}{\Gamma(1+3 \alpha)}(x-a)^{\alpha}\right)(x-a)^{2 \alpha}\|g\|_{[a, x], \infty}\right. \\
& \left.+\left(\frac{\Gamma(1+\alpha)}{\Gamma(1+2 \alpha)}-\frac{\Gamma(1+2 \alpha)}{\Gamma(1+3 \alpha)}\right)(b-x)^{3 \alpha}\|g\|_{[x, b], \infty}\right] \frac{\left|f^{(\alpha)}(a)\right|}{(b-a)^{\alpha}} \\
& +\left[\left(\frac{\Gamma(1+2 \alpha)}{\Gamma(1+3 \alpha)}-\frac{\Gamma(1+\alpha)}{\Gamma(1+2 \alpha)}\right)(x-a)^{3 \alpha}\|g\|_{[a, x], \infty}\right. \\
& \left.+\left(\frac{\Gamma(1+\alpha)}{\Gamma(1+2 \alpha)}(x-a)^{\alpha}+\frac{\Gamma(1+2 \alpha)}{\Gamma(1+3 \alpha)}(b-x)^{\alpha}\right)(b-x)^{2 \alpha}\|g\|_{[x, b], \infty}\right] \frac{\left|f^{(\alpha)}(b)\right|}{(b-a)^{\alpha}}
\end{aligned}
$$




$$
\begin{aligned}
\leq & \frac{\|g\|_{[a, b], \infty}}{(b-a)^{\alpha}}\left(\left[\frac{\Gamma(1+\alpha)}{\Gamma(1+2 \alpha)}\left[(x-b)^{\alpha}(x-a)^{2 \alpha}+(b-x)^{3 \alpha}\right]+\frac{\Gamma(2+\alpha)}{\Gamma(2+2 \alpha)}\left[(x-a)^{3 \alpha}-(b-x)^{3 \alpha}\right]\right]\left|f^{(\alpha)}(a)\right|\right. \\
& \left.\times\left[\frac{\Gamma(1+\alpha)}{\Gamma(1+2 \alpha)}\left[(x-a)^{\alpha}(b-x)^{2 \alpha}-(x-a)^{3 \alpha}\right]+\frac{\Gamma(2+\alpha)}{\Gamma(2+2 \alpha)}\left[(x-a)^{3 \alpha}+(b-x)^{3 \alpha}\right]\right]\left|f^{(\alpha)}(b)\right|\right) .
\end{aligned}
$$

Proof. Let $x \in[a, b]$. Using Lemma 15 and the generalized convexty of $\left|f^{(\alpha)}\right|$, we have

$$
\begin{aligned}
& \left|f(a)_{a} I_{x}^{(\alpha)} g(s)+f(b)_{x} I_{b}^{(\alpha)} g(s)-{ }_{a} I_{b}^{(\alpha)} f(s) g(s)\right| \\
& \leq \frac{1}{\Gamma(1+\alpha)}\left(\int_{a}^{x}\left|\frac{1}{\Gamma(1+\alpha)} \int_{x}^{t} g(s)(d s)^{\alpha}\right|\left|f^{(\alpha)}(t)\right|(d t)^{\alpha}+\int_{x}^{b}\left|\frac{1}{\Gamma(1+\alpha)} \int_{x}^{t} g(s)(d s)^{\alpha}\right|\left|f^{(\alpha)}(t)\right|(d t)^{\alpha}\right) \\
& \leq \frac{1}{\Gamma(1+\alpha)}\left(\|g\|_{[a, x], \infty} \int_{a}^{x}(x-t)^{\alpha}\left|f^{(\alpha)}(t)\right|(d t)^{\alpha}+\|g\|_{[x, b], \infty} \int_{x}^{b}(t-x)^{\alpha}\left|f^{(\alpha)}(t)\right|(d t)^{\alpha}\right) \\
& =\frac{\|g\|_{[a, x], \infty}}{\Gamma(1+\alpha)} \int_{a}^{x}(x-t)^{\alpha}\left|f^{(\alpha)}\left(\frac{b-t}{b-a} a+\frac{t-a}{b-a} b\right)\right|(d t)^{\alpha}+\frac{\|g\|_{[x, b], \infty}}{\Gamma(1+\alpha)} \int_{x}^{b}(t-x)^{\alpha}\left|f^{(\alpha)}\left(\frac{b-t}{b-a} a+\frac{t-a}{b-a} b\right)\right|(d t)^{\alpha} \\
& \leq \frac{1}{\Gamma(1+\alpha)}\left[\frac{\|g\|_{[a, x], \infty}}{(b-a)^{\alpha}}\left(\left|f^{(\alpha)}(a)\right| \int_{a}^{x}(x-t)^{\alpha}(b-t)^{\alpha}(d t)^{\alpha}+\left|f^{(\alpha)}(b)\right| \int_{a}^{x}(x-t)^{\alpha}(t-a)^{\alpha}(d t)^{\alpha}\right)\right. \\
& \left.+\frac{\|g\|_{[x, b], \infty}}{(b-a)^{\alpha}}\left(\left|f^{(\alpha)}(a)\right| \int_{x}^{b}(t-x)^{\alpha}(b-t)^{\alpha}(d t)^{\alpha}+\left|f^{(\alpha)}(b)\right| \int_{x}^{b}(t-x)^{\alpha}(t-a)^{\alpha}(d t)^{\alpha}\right)\right] .
\end{aligned}
$$

Now by variable exchange method, we have

$$
\begin{gathered}
\frac{1}{\Gamma(1+\alpha)} \int_{a}^{x}(x-t)^{\alpha}(b-t)^{\alpha}(d t)^{\alpha}=\left[\frac{\Gamma(1+\alpha)}{\Gamma(1+2 \alpha)}(b-x)^{\alpha}+\frac{\Gamma(1+2 \alpha)}{\Gamma(1+3 \alpha)}(x-a)^{\alpha}\right](x-a)^{2 \alpha}, \\
\frac{1}{\Gamma(1+\alpha)} \int_{a}^{x}(x-t)^{\alpha}(t-a)^{\alpha}(d t)^{\alpha}=\left[\frac{\Gamma(1+2 \alpha)}{\Gamma(1+3 \alpha)}-\frac{\Gamma(1+\alpha)}{\Gamma(1+2 \alpha)}\right](x-a)^{3 \alpha}, \\
\frac{1}{\Gamma(1+\alpha)} \int_{x}^{b}(t-x)^{\alpha}(b-t)^{\alpha}(d t)^{\alpha}=\left[\frac{\Gamma(1+\alpha)}{\Gamma(1+2 \alpha)}-\frac{\Gamma(1+2 \alpha)}{\Gamma(1+3 \alpha)}\right](b-x)^{3 \alpha},
\end{gathered}
$$

and

$$
\frac{1}{\Gamma(1+\alpha)} \int_{x}^{b}(t-x)^{\alpha}(t-a)^{\alpha}(d t)^{\alpha}=\left[\frac{\Gamma(1+\alpha)}{\Gamma(1+2 \alpha)}(x-a)^{\alpha}+\frac{\Gamma(1+2 \alpha)}{\Gamma(1+3 \alpha)}(b-x)^{\alpha}\right](b-x)^{2 \alpha} .
$$

Using (7) - (10) in (6) we obtain

$$
\begin{aligned}
& \left|f(a)_{a} I_{x}^{(\alpha)} g(x)+f(b)_{x} I_{b}^{(\alpha)} g(x)-{ }_{a} I_{b}^{(\alpha)} f(x) g(x)\right| \\
& \leq\left[\left(\frac{\Gamma(1+\alpha)}{\Gamma(1+2 \alpha)}(b-x)^{\alpha}+\frac{\Gamma(1+2 \alpha)}{\Gamma(1+3 \alpha)}(x-a)^{\alpha}\right)(x-a)^{2 \alpha}\|g\|_{[a, x], \infty}\right. \\
& \left.+\left(\frac{\Gamma(1+\alpha)}{\Gamma(1+2 \alpha)}-\frac{\Gamma(1+2 \alpha)}{\Gamma(1+3 \alpha)}\right)(b-x)^{3 \alpha}\|g\|_{[x, b], \infty}\right] \frac{\left|f^{(\alpha)}(a)\right|}{(b-a)^{\alpha}} \\
& +\left[\left(\frac{\Gamma(1+2 \alpha)}{\Gamma(1+3 \alpha)}-\frac{\Gamma(1+\alpha)}{\Gamma(1+2 \alpha)}\right)(x-a)^{3 \alpha}\|g\|_{[a, x], \infty}\right. \\
& \left.+\left(\frac{\Gamma(1+\alpha)}{\Gamma(1+2 \alpha)}(x-a)^{\alpha}+\frac{\Gamma(1+2 \alpha)}{\Gamma(1+3 \alpha)}(b-x)^{\alpha}\right)(b-x)^{2 \alpha}\|g\|_{[x, b], \infty}\right] \frac{\left|f^{(\alpha)}(b)\right|}{(b-a)^{\alpha}} .
\end{aligned}
$$

Since $\|g\|_{[a, x], \infty},\|g\|_{[x, b], \infty} \leq\|g\|_{[a, b], \infty}$, we have

$$
\begin{aligned}
& \left|f(a)_{a} I_{x}^{(\alpha)} g(x)+f(b)_{x} I_{b}^{(\alpha)} g(x)-{ }_{a} I_{b}^{(\alpha)} f(x) g(x)\right| \\
& \leq\left[\left(\frac{\Gamma(1+\alpha)}{\Gamma(1+2 \alpha)}(b-x)^{\alpha}+\frac{\Gamma(1+2 \alpha)}{\Gamma(1+3 \alpha)}(x-a)^{\alpha}\right)(x-a)^{2 \alpha}\|g\|_{[a, x], \infty}\right.
\end{aligned}
$$




$$
\begin{aligned}
& \left.+\left(\frac{\Gamma(1+\alpha)}{\Gamma(1+2 \alpha)}-\frac{\Gamma(1+2 \alpha)}{\Gamma(1+3 \alpha)}\right)(b-x)^{3 \alpha}\|g\|_{[x, b], \infty}\right] \frac{\left|f^{(\alpha)}(a)\right|}{(b-a)^{\alpha}} \\
& +\left[\left(\frac{\Gamma(1+2 \alpha)}{\Gamma(1+3 \alpha)}-\frac{\Gamma(1+\alpha)}{\Gamma(1+2 \alpha)}\right)(x-a)^{3 \alpha}\|g\|_{[a, x], \infty}\right. \\
& \left.+\left(\frac{\Gamma(1+\alpha)}{\Gamma(1+2 \alpha)}(x-a)^{\alpha}+\frac{\Gamma(1+2 \alpha)}{\Gamma(1+3 \alpha)}(b-x)^{\alpha}\right)(b-x)^{2 \alpha}\|g\|_{[x, b], \infty}\right] \frac{\left|f^{(\alpha)}(b)\right|}{(b-a)^{\alpha}} \\
& \leq \frac{\|g\|_{[a, b], \infty}}{(b-a)^{\alpha}}\left(\left[\frac{\Gamma(1+\alpha)}{\Gamma(1+2 \alpha)}\left[(x-b)^{\alpha}(x-a)^{2 \alpha}+(b-x)^{3 \alpha}\right]\right.\right. \\
& \left.+\frac{\Gamma(2+\alpha)}{\Gamma(2+2 \alpha)}\left[(x-a)^{3 \alpha}-(b-x)^{3 \alpha}\right]\right]\left|f^{(\alpha)}(a)\right|\left[\frac{\Gamma(1+\alpha)}{\Gamma(1+2 \alpha)}\left[(x-a)^{\alpha}(b-x)^{2 \alpha}-(x-a)^{3 \alpha}\right]\right. \\
& \left.\left.+\frac{\Gamma(2+\alpha)}{\Gamma(2+2 \alpha)}\left[(x-a)^{3 \alpha}+(b-x)^{3 \alpha}\right]\right]\left|f^{(\alpha)}(b)\right|\right) .
\end{aligned}
$$

Hence, the proof is completed.

Using Theorem 16, we have the following corollaries which are connected with the right-hand side of Fejér inequality.

Corollary 17. Let $0 \leq \lambda \leq 1$ and $x=\lambda a+(1-\lambda) b$ in Theorem 16. Then we have

$$
\begin{aligned}
& \left|f(a)_{a} I_{(\lambda a+(1-\lambda) b)}^{(\alpha)} g(s)+f(b)(\lambda a+(1-\lambda) b) I_{b}^{(\alpha)} g(s)-{ }_{a} I_{b}^{(\alpha)} f(s) g(s)\right| \\
\leq \quad & {\left[\left(\frac{\Gamma(1+\alpha)}{\Gamma(1+2 \alpha)} \lambda^{\alpha}+\frac{\Gamma(1+2 \alpha)}{\Gamma(1+3 \alpha)}(1-\lambda)^{\alpha}\right)(1-\lambda)^{2 \alpha}\|g\|_{[a, x], \infty}\right.} \\
& \left.+\left(\frac{\Gamma(1+\alpha)}{\Gamma(1+2 \alpha)}-\frac{\Gamma(1+2 \alpha)}{\Gamma(1+3 \alpha)}\right) \lambda^{3 \alpha}\|g\|_{[x, b], \infty}\right](b-a)^{2 \alpha}\left|f^{(\alpha)}(a)\right| \\
+ & {\left[\left(\frac{\Gamma(1+2 \alpha)}{\Gamma(1+3 \alpha)}-\frac{\Gamma(1+\alpha)}{\Gamma(1+2 \alpha)}\right)(1-\lambda)^{3 \alpha}\|g\|_{[a, x], \infty}\right.} \\
& \left.+\left(\frac{\Gamma(1+\alpha)}{\Gamma(1+2 \alpha)}(1-\lambda)^{\alpha}+\frac{\Gamma(1+2 \alpha)}{\Gamma(1+3 \alpha)} \lambda^{\alpha}\right) \lambda^{2 \alpha}\|g\|_{[x, b], \infty}\right](b-a)^{2 \alpha}\left|f^{(\alpha)}(b)\right| \\
\leq \quad & \left(\left[\frac{\Gamma(1+\alpha)}{\Gamma(1+2 \alpha)}\left(\lambda^{\alpha}(1-\lambda)^{2 \alpha}+\lambda^{3 \alpha}\right)+\frac{\Gamma(1+2 \alpha)}{\Gamma(1+3 \alpha)}\left((1-\lambda)^{3 \alpha}-\lambda^{3 \alpha}\right)\right]\left|f^{(\alpha)}(a)\right|\right. \\
+ & {\left.\left[\frac{\Gamma(1+\alpha)}{\Gamma(1+2 \alpha)}\left((1-\lambda)^{\alpha} \lambda^{2 \alpha}-(1-\lambda)^{3 \alpha}\right)+\frac{\Gamma(1+2 \alpha)}{\Gamma(1+3 \alpha)}\left((1-\lambda)^{3 \alpha}+\lambda^{3 \alpha}\right)\right]\left|f^{(\alpha)}(b)\right|\right)(b-a)^{2 \alpha}\|g\|_{[a, b], \infty} . }
\end{aligned}
$$

Corollary 18. Let $g:[a, b] \rightarrow R^{\alpha}$ be a symmetric to $(a+b) / 2$ and $\lambda=1 / 2$ in Corollary 17 . Then we have the inequality

$$
\begin{aligned}
& \left|\frac{f(a)+f(b)}{2^{\alpha}}{ }_{a} I_{b}^{(\alpha)} g(s)-{ }_{a} I_{b}^{(\alpha)} f(s) g(s)\right| \leq\left[\frac{\Gamma(1+\alpha)}{\Gamma(1+2 \alpha)}\left(\|g\|_{[a, x], \infty}+\|g\|_{[x, b], \infty}\right)\right. \\
& \left.+\frac{\Gamma(1+2 \alpha)}{\Gamma(1+3 \alpha)}\left(\|g\|_{[a, x], \infty}-\|g\|_{[x, b], \infty}\right)\right] \frac{(b-a)^{2 \alpha}\left|f^{(\alpha)}(a)\right|}{2^{3 \alpha}}+\left[\frac{\Gamma(1+\alpha)}{\Gamma(1+2 \alpha)}\left(\|g\|_{[x, b], \infty}-\|g\|_{[a, x], \infty}\right)\right. \\
& \left.+\frac{\Gamma(1+2 \alpha)}{\Gamma(1+3 \alpha)}\left(\|g\|_{[a, x], \infty}+\|g\|_{[x, b], \infty}\right)\right] \frac{(b-a)^{2 \alpha}\left|f^{(\alpha)}(b)\right|}{2^{3 \alpha}} \\
& \leq\left(\frac{\Gamma(1+\alpha)}{\Gamma(1+2 \alpha)}\left|f^{(\alpha)}(a)\right|+\frac{\Gamma(1+2 \alpha)}{\Gamma(1+3 \alpha)}\left|f^{(\alpha)}(b)\right|\right) \frac{(b-a)^{2 \alpha}\|g\|_{[a, b], \infty}}{2^{2 \alpha}}
\end{aligned}
$$

which is the "weighted trapezoid" inequality provided that $\left|f^{(\alpha)}\right|$ is generalized convex on $[a, b]$.

Proof. Using the symmetry of $g$, we have the following identity

$$
f(a)_{a} I_{\frac{a+b}{2}}^{(\alpha)} g(x)+f(b)_{\frac{a+b}{2}} I_{b}^{(\alpha)} g(x)-{ }_{a} I_{b}^{(\alpha)} f(x) g(x)=\frac{f(a)+f(b)}{2^{\alpha}}{ }_{a} I_{b}^{(\alpha)} g(x)-{ }_{a} I_{b}^{(\alpha)} f(x) g(x) .
$$

From this identity and Corollary 17, we have the inequality (11). This completes the proof. 
Remark 2. If we choose $g(t)=1^{\alpha}$ on $[a, b]$, then we obtain following the inequality from (11)

$$
\left|\frac{f(a)+f(b)}{2^{\alpha} \Gamma(1+\alpha)}(b-a)^{\alpha}-{ }_{a} I_{b}^{(\alpha)} f(s)\right| \leq\left(\frac{\Gamma(1+\alpha)}{\Gamma(1+2 \alpha)}\left|f^{(\alpha)}(a)\right|+\frac{\Gamma(1+2 \alpha)}{\Gamma(1+3 \alpha)}\left|f^{(\alpha)}(b)\right|\right) \frac{(b-a)^{2 \alpha}}{2^{2 \alpha}}
$$

Remark 3. Let $f(x) \in D_{\alpha}[a, b]$ be a generalized convex function on $[a, b]$ with $a<b$ and let $g(x) \in C_{\alpha}[a, b]$ with symmetric to $(a+b) / 2$. Then (11) is an error bound of the second inequality in generalized Fejer inequality

$$
\begin{aligned}
0 \leq & \frac{f(a)+f(b)}{2^{\alpha}}{ }_{a} I_{b}^{(\alpha)} g(s)-{ }_{a} I_{b}^{(\alpha)} f(s) g(s) \\
\leq & {\left[\frac{\Gamma(1+\alpha)}{\Gamma(1+2 \alpha)}\left(\|g\|_{[a, x], \infty}+\|g\|_{[x, b], \infty}\right)+\frac{\Gamma(1+2 \alpha)}{\Gamma(1+3 \alpha)}\left(\|g\|_{[a, x], \infty}-\|g\|_{[x, b], \infty}\right)\right] \frac{(b-a)^{2 \alpha}\left|f^{(\alpha)}(a)\right|}{2^{3 \alpha}} } \\
& +\left[\frac{\Gamma(1+\alpha)}{\Gamma(1+2 \alpha)}\left(\|g\|_{[x, b], \infty}-\|g\|_{[a, x], \infty}\right)+\frac{\Gamma(1+2 \alpha)}{\Gamma(1+3 \alpha)}\left(\|g\|_{[a, x], \infty}+\|g\|_{[x, b], \infty}\right)\right] \frac{(b-a)^{2 \alpha}\left|f^{(\alpha)}(b)\right|}{2^{3 \alpha}} \\
\leq & \left(\frac{\Gamma(1+\alpha)}{\Gamma(1+2 \alpha)}\left|f^{(\alpha)}(a)\right|+\frac{\Gamma(1+2 \alpha)}{\Gamma(1+3 \alpha)}\left|f^{(\alpha)}(b)\right|\right) \frac{(b-a)^{2 \alpha}\|g\|_{[a, b], \infty}}{2^{2 \alpha}}
\end{aligned}
$$

provided that $\left|f^{(\alpha)}\right|$ is generalized convex on $[a, b]$ and $f^{(\alpha)} \in I_{x}^{(\alpha)}[a, b]$.

Theorem 19. Let $f(x) \in D_{\alpha}[a, b]$ with $a<b$ and let $f^{(\alpha)} \in I_{x}^{(\alpha)}[a, b], g(x) \in C_{\alpha}[a, b]$. If $\left|f^{(\alpha)}\right|^{p /(p-1)}$ is generalized convex on $[a, b]$ with $p>1$, then, for all $x \in[a, b]$, the following inequality holds:

$$
\begin{aligned}
& \left|f(a)_{a} I_{x}^{(\alpha)} g(s)+f(b)_{x} I_{b}^{(\alpha)} g(s)-{ }_{a} I_{b}^{(\alpha)} f(s) g(s)\right| \\
\leq & \frac{(b-a)^{\alpha \frac{p-1}{p}}\|g\|_{[a, b], \infty}}{\Gamma(1+\alpha)}\left(\frac{\Gamma(1+p \alpha)}{\Gamma(1+(p+1) \alpha)}\right)^{\frac{1}{p}}\left((x-a)^{(p+1) \alpha}-(b-x)^{(p+1) \alpha}\right)^{\frac{1}{p}} \\
& \cdot\left(\frac{\Gamma(1+\alpha)}{\Gamma(1+2 \alpha)}\right)^{\frac{p-1}{p}}\left[\left|f^{(\alpha)}(a)\right|^{\frac{p}{p-1}}+\left|f^{(\alpha)}(b)\right|^{\frac{p}{p-1}}\right]^{\frac{p-1}{p}} \cdot
\end{aligned}
$$

Proof. Let $x \in[a, b]$, Lemma 15, generalized Hölder's inequality and the generalized convexity of $\left|f^{(\alpha)}\right|^{p /(p-1)}$, it follows that

$$
\begin{aligned}
& \left|f(a)_{a} I_{x}^{(\alpha)} g(s)+f(b)_{x} I_{b}^{(\alpha)} g(s)-{ }_{a} I_{b}^{(\alpha)} f(s) g(s)\right| \\
& \leq\left[\frac{1}{\Gamma(1+\alpha)} \int_{a}^{b}\left|\frac{1}{\Gamma(1+\alpha)} \int_{x}^{t} g(s)(d s)^{\alpha}\right|^{p}(d t)^{\alpha}\right]^{\frac{1}{p}}\left[\frac{1}{\Gamma(1+\alpha)} \int_{a}^{b} \mid f^{(\alpha)}(t)^{\frac{p}{p-1}}(d t)^{\alpha}\right]^{\frac{p-1}{p}} \\
& \leq \frac{\|g\|_{[a, b], \infty}}{\Gamma(1+\alpha)}\left[\frac{1}{\Gamma(1+\alpha)} \int_{a}^{b}|t-x|^{\alpha p}(d t)^{\alpha}\right]^{\frac{1}{p}} \\
& \times\left[\frac{1}{\Gamma(1+\alpha)} \int_{a}^{b}\left(\frac{(b-t)^{\alpha}}{(b-a)^{\alpha}}\left|f^{(\alpha)}(a)\right|^{\frac{p}{p-1}}+\frac{(t-a)^{\alpha}}{(b-a)^{\alpha}}\left|f^{(\alpha)}(b)\right|^{\frac{p}{p-1}}\right)(d t)^{\alpha}\right]^{\frac{p-1}{p}} \\
& =\frac{\|g\|_{[a, b], \infty}}{\Gamma(1+\alpha)}\left[\frac{1}{\Gamma(1+\alpha)} \int_{a}^{x}(x-t)^{\alpha p}(d t)^{\alpha}+\frac{1}{\Gamma(1+\alpha)} \int_{x}^{b}(t-x)^{\alpha p}(d t)^{\alpha}\right]^{\frac{1}{p}} \\
& \times\left[\frac{\left|f^{(\alpha)}(a)\right|^{\frac{p}{p-1}}}{(b-a)^{\alpha}} \frac{1}{\Gamma(1+\alpha)} \int_{a}^{b}(b-t)^{\alpha}(d t)^{\alpha}+\frac{\left|f^{(\alpha)}(b)\right|^{\frac{p}{p-1}}}{(b-a)^{\alpha}} \frac{1}{\Gamma(1+\alpha)} \int_{a}^{b}(t-a)^{\alpha}(d t)^{\alpha}\right]^{\frac{p-1}{p}} \\
& =\frac{\|g\|_{[a, b], \infty}}{\Gamma(1+\alpha)}\left[\frac{\Gamma(1+p \alpha)}{\Gamma(1+(p+1) \alpha)}\left((x-a)^{(p+1) \alpha}+(b-x)^{(p+1) \alpha}\right)\right]^{\frac{1}{p}}
\end{aligned}
$$




$$
\begin{aligned}
& \times\left[\frac{\left|f^{(\alpha)}(a)\right|^{\frac{p}{p-1}}}{(b-a)^{\alpha}} \frac{\Gamma(1+\alpha)}{\Gamma(1+2 \alpha)}(b-a)^{2 \alpha}+\frac{\left|f^{(\alpha)}(b)\right|^{\frac{p}{p-1}}}{(b-a)^{\alpha}} \frac{\Gamma(1+\alpha)}{\Gamma(1+2 \alpha)}(b-a)^{2 \alpha}\right]^{\frac{p-1}{p}} \\
= & \frac{(b-a)^{\alpha \frac{p-1}{p}}\|g\|_{[a, b], \infty}}{\Gamma(1+\alpha)}\left(\frac{\Gamma(1+p \alpha)}{\Gamma(1+(p+1) \alpha)}\right)^{\frac{1}{p}}\left((x-a)^{(p+1) \alpha}+(b-x)^{(p+1) \alpha}\right)^{\frac{1}{p}} \\
& \times\left(\frac{\Gamma(1+\alpha)}{\Gamma(1+2 \alpha)}\right)^{\frac{p-1}{p}}\left[\left|f^{(\alpha)}(a)\right|^{\frac{p}{p-1}}+\left|f^{(\alpha)}(b)\right|^{\frac{p}{p-1}}\right]^{\frac{p-1}{p}}
\end{aligned}
$$

and this completes the proof.

Using Theorem 19, we have the following corollaries which are connected with the right-hand side of Fejér inequality.

Corollary 20. Let $0 \leq \lambda \leq 1$ and $x=\lambda a+(1-\lambda) b$ in Theorem 5. Then we have the inequality

$$
\begin{aligned}
& \left|f(a)_{a} I_{\lambda a+(1-\lambda) b}^{(\alpha)} g(s)+f(b)_{\lambda a+(1-\lambda) b} I_{b}^{(\alpha)} g(s)-{ }_{a} I_{b}^{(\alpha)} f(s) g(s)\right| \\
\leq & \frac{(b-a)^{2 \alpha}\|g\|_{[a, b], \infty}}{\Gamma(1+\alpha)}\left(\frac{\Gamma(1+p \alpha)}{\Gamma(1+(p+1) \alpha)}\right)^{\frac{1}{p}}\left((1-\lambda)^{(p+1) \alpha}+\lambda^{(p+1) \alpha}\right)^{\frac{1}{p}} \\
& \times\left(\frac{\Gamma(1+\alpha)}{\Gamma(1+2 \alpha)}\right)^{\frac{p-1}{p}}\left[\left|f^{(\alpha)}(a)\right|^{\frac{p}{p-1}}+\left|f^{(\alpha)}(b)\right|^{\frac{p}{p-1}}\right]^{\frac{p-1}{p}} .
\end{aligned}
$$

Corollary 21. Let $g:[a, b] \rightarrow R^{\alpha}$ be a symmetric to $(a+b) / 2$ and $\lambda=1 / 2$ in Corollary 20. Then we have the inequality

$$
\begin{aligned}
& \left|\frac{f(a)+f(b)}{2^{\alpha}} a_{b}^{(\alpha)} g(s)-{ }_{a} I_{b}^{(\alpha)} f(s) g(s)\right| \leq \frac{(b-a)^{2 \alpha}\|g\|_{[a, b], \infty}}{2^{\alpha} \Gamma(1+\alpha)}\left(\frac{\Gamma(1+p \alpha)}{\Gamma(1+(p+1) \alpha)}\right)^{\frac{1}{p}} \\
& \times\left(\frac{\Gamma(1+\alpha)}{\Gamma(1+2 \alpha)}\right)^{\frac{p-1}{p}}\left[\left|f^{(\alpha)}(a)\right|^{\frac{p}{p-1}}+\left|f^{(\alpha)}(b)\right|^{\frac{p}{p-1}}\right]^{\frac{p-1}{p}} .
\end{aligned}
$$

which is the "weighted trapezoid" inequality provided that $\left|f^{(\alpha)}\right|^{p /(p-1)}$ is generalized convex on $[a, b]$ and $f^{(\alpha)} \in$ $I_{x}^{(\alpha)}[a, b]$. where $p>1$.

Remark 4. If we choose $g(t)=1^{\alpha}$ on $[a, b]$, then we obtain following the inequality from (15)

$$
\begin{aligned}
& \left|\frac{f(a)+f(b)}{2^{\alpha} \Gamma(1+\alpha)}(b-a)^{\alpha}-{ }_{a} I_{b}^{(\alpha)} f(s)\right| \leq \frac{(b-a)^{2 \alpha}}{2^{\alpha} \Gamma(1+\alpha)}\left(\frac{\Gamma(1+p \alpha)}{\Gamma(1+(p+1) \alpha)}\right)^{\frac{1}{p}} \\
& \times\left(\frac{\Gamma(1+\alpha)}{\Gamma(1+2 \alpha)}\right)^{\frac{p-1}{p}}\left[\left|f^{(\alpha)}(a)\right|^{\frac{p}{p-1}}+\left|f^{(\alpha)}(b)\right|^{\frac{p}{p-1}}\right]^{\frac{p-1}{p}} .
\end{aligned}
$$

Remark 5. Let $p>1, f(x) \in D_{\alpha}[a, b]$ be a generalized convex and let $g(x) \in C_{\alpha}[a, b]$ with symmetric to $(a+b) / 2$. Using Corollary 21, we obtain an error bound of the second inequality in generalized Fejèr inequality

$$
\begin{aligned}
0 & \leq \frac{f(a)+f(b)}{2^{\alpha}}{ }_{a} I_{b}^{(\alpha)} g(s)-{ }_{a} I_{b}^{(\alpha)} f(s) g(s) \\
& \leq \frac{(b-a)^{2 \alpha}\|g\|_{[a, b], \infty}}{2^{\alpha} \Gamma(1+\alpha)}\left(\frac{\Gamma(1+p \alpha)}{\Gamma(1+(p+1) \alpha)}\right)^{\frac{1}{p}} \times\left(\frac{\Gamma(1+\alpha)}{\Gamma(1+2 \alpha)}\right)^{\frac{p-1}{p}}\left[\left|f^{(\alpha)}(a)\right|^{\frac{p}{p-1}}+\left|f^{(\alpha)}(b)\right|^{\frac{p}{p-1}}\right]^{\frac{p-1}{p}}
\end{aligned}
$$

provided that $\left|f^{(\alpha)}\right|^{p /(p-1)}$ is generalized convex on $[a, b]$ and $f^{(\alpha)} \in I_{x}^{(\alpha)}[a, b]$. 


\section{Applications to Weighted Trapezoided Formula}

Throughout this section, let $g(x) \in C_{\alpha}[a, b], f \in I_{x}^{(\alpha)}[a, b]$ and let $I_{n}: a=x_{0}<x_{1}<\ldots<x_{n}=b$ be a partition of $[a, b]$ and $l_{i}=x_{i+1}-x_{i}(i=0,1, \ldots, n-1)$. Define

$$
\begin{aligned}
T_{\alpha}\left(f, I_{n}\right) & =\frac{1}{\Gamma(1+\alpha)} \sum_{i=0}^{n-1} \frac{f\left(x_{i}\right)+f\left(x_{i+1}\right)}{2^{\alpha}} l_{i}^{\alpha}, \\
E_{\alpha}\left(f, I_{n}\right) & ={ }_{a} I_{b}^{(\alpha)} f(x)-T_{\alpha}\left(f, I_{n}\right), \\
T_{\alpha}\left(f, g, I_{n}\right) & =\frac{1}{\Gamma(1+\alpha)} \sum_{i=0}^{n-1}\left[f\left(x_{i}\right) \int_{x_{i}}^{\frac{x_{i}+x_{i+1}}{2}} g(s)(d s)^{\alpha}+f\left(x_{i+1}\right) \int_{\frac{x_{i}+x_{i+1}}{2}}^{x_{i+1}} g(s)(d s)^{\alpha}\right], \\
E_{\alpha}\left(f, g, I_{n}\right) & =a I_{b}^{(\alpha)} f(x) g(x)-T_{\alpha}\left(f, g, I_{n}\right) .
\end{aligned}
$$

Proposition 22. Let $f(x) \in D_{\alpha}[a, b]$ with $a<b$ and let $f^{(\alpha)} \in I_{x}^{(\alpha)}[a, b]$. Let $\left|f^{(\alpha)}\right|$ is a generalized convex on $[a, b]$. Then we have

$$
\begin{aligned}
\left|E_{\alpha}\left(f, I_{n}\right)\right| & \leq \frac{1}{2^{2 \alpha}} \sum_{i=0}^{n-1}\left(\frac{\Gamma(1+\alpha)}{\Gamma(1+2 \alpha)}\left|f^{(\alpha)}\left(x_{i}\right)\right|+\frac{\Gamma(1+2 \alpha)}{\Gamma(1+3 \alpha)}\left|f^{(\alpha)}\left(x_{i+1}\right)\right|\right) l_{i}^{2 \alpha} \\
& \leq \frac{\max \left\{\frac{\Gamma(1+\alpha)}{\Gamma(1+2 \alpha)}\left|f^{(\alpha)}(a)\right|+\frac{\Gamma(1+2 \alpha)}{\Gamma(1+3 \alpha)}\left|f^{(\alpha)}(b)\right|\right\}}{2^{2 \alpha}} \sum_{i=0}^{n-1} l_{i}^{2 \alpha} .
\end{aligned}
$$

Proof. Using (12) the following inequality holds

$$
\left|\frac{f\left(x_{i}\right)+f\left(x_{i+1}\right)}{2^{\alpha} \Gamma(1+\alpha)} l_{i}^{\alpha}-x_{i} I_{x_{i+1}}^{(\alpha)} f(s)\right| \leq\left(\frac{\Gamma(1+\alpha)}{\Gamma(1+2 \alpha)}\left|f^{(\alpha)}\left(x_{i}\right)\right|+\frac{\Gamma(1+2 \alpha)}{\Gamma(1+3 \alpha)}\left|f^{(\alpha)}\left(x_{i+1}\right)\right|\right) \frac{l_{i}^{2 \alpha}}{2^{2 \alpha}} .
$$

Hence in the trapezoidal case

$$
\begin{aligned}
\left|T_{\alpha}\left(f, I_{n}\right)-{ }_{a} I_{b}^{(\alpha)} f(x)\right| & =\left|\sum_{i=0}^{n-1}\left\{\frac{f\left(x_{i}\right)+f\left(x_{i+1}\right)}{2^{\alpha} \Gamma(1+\alpha)} l_{i}^{\alpha}-\frac{1}{\Gamma(1+\alpha)} \int_{x_{i}}^{x_{i+1}} f(s)(d s)^{\alpha}\right\}\right| \\
& \sum_{i=0}^{n-1}\left|\frac{f\left(x_{i}\right)+f\left(x_{i+1}\right)}{2^{\alpha} \Gamma(1+\alpha)} l_{i}^{\alpha}-\frac{1}{\Gamma(1+\alpha)} \int_{x_{i}}^{x_{i+1}} f(s)(d s)^{\alpha}\right| \\
& \leq \sum_{i=0}^{n-1}\left(\frac{\Gamma(1+\alpha)}{\Gamma(1+2 \alpha)}\left|f^{(\alpha)}\left(x_{i}\right)\right|+\frac{\Gamma(1+2 \alpha)}{\Gamma(1+3 \alpha)}\left|f^{(\alpha)}\left(x_{i+1}\right)\right|\right) \frac{l_{i}^{2 \alpha}}{2^{2 \alpha}} \\
& =\frac{1}{2^{2 \alpha}} \sum_{i=0}^{n-1}\left(\frac{\Gamma(1+\alpha)}{\Gamma(1+2 \alpha)}\left|f^{(\alpha)}\left(x_{i}\right)\right|+\frac{\Gamma(1+2 \alpha)}{\Gamma(1+3 \alpha)}\left|f^{(\alpha)}\left(x_{i+1}\right)\right|\right) l_{i}^{2 \alpha} \\
& \leq \frac{\max \left\{\frac{\Gamma(1+\alpha)}{\Gamma(1+2 \alpha)}\left|f^{(\alpha)}(a)\right|+\frac{\Gamma(1+2 \alpha)}{\Gamma(1+3 \alpha)}\left|f^{(\alpha)}(b)\right|\right\}}{2^{2 \alpha}} \sum_{i=0}^{n-1} l_{i}^{2 \alpha} .
\end{aligned}
$$

This completes the proof.

Proposition 23. Let $f(x) \in D_{\alpha}[a, b]$ with $a<b$ and let $f^{(\alpha)} \in I_{x}^{(\alpha)}[a, b]$. Let $\left|f^{(\alpha)}\right|^{\frac{p}{p-1}}$ is a generalized convex on $[a, b]$ with $p>1$. Then we have

$$
\begin{aligned}
\left|E_{\alpha}\left(f, I_{n}\right)\right| \leq & \frac{1}{2^{\alpha} \Gamma(1+\alpha)}\left(\frac{\Gamma(1+p \alpha)}{\Gamma(1+(p+1) \alpha)}\right)^{\frac{1}{p}} \\
& \times\left(\frac{\Gamma(1+\alpha)}{\Gamma(1+2 \alpha)}\right)^{\frac{p-1}{p}} \sum_{i=0}^{n-1}\left[\left|f^{(\alpha)}\left(x_{i}\right)\right|^{\frac{p}{p-1}}+\left|f^{(\alpha)}\left(x_{i+1}\right)\right|^{\frac{p}{p-1}}\right]^{\frac{p-1}{p}} l_{i}^{2 \alpha} \\
\leq & \frac{1}{2^{\alpha} \Gamma(1+\alpha)}\left(\frac{\Gamma(1+p \alpha)}{\Gamma(1+(p+1) \alpha)}\right)^{\frac{1}{p}}\left(\frac{\Gamma(1+\alpha)}{\Gamma(1+2 \alpha)}\right)^{\frac{p-1}{p}} \max \left\{\left|f^{(\alpha)}\left(x_{i}\right)\right|,\left|f^{(\alpha)}\left(x_{i+1}\right)\right|\right\} \sum_{i=0}^{n-1} l_{i}^{2 \alpha}
\end{aligned}
$$


Proof. Using (16) the following inequality holds

$$
\begin{aligned}
& \left|\frac{f\left(x_{i}\right)+f\left(x_{i+1}\right)}{2^{\alpha} \Gamma(1+\alpha)} l_{i}^{\alpha}-x_{i} I_{x_{i+1}}^{(\alpha)} f(s)\right| \\
\leq & \frac{l_{i}^{2 \alpha}}{2^{\alpha} \Gamma(1+\alpha)}\left(\frac{\Gamma(1+p \alpha)}{\Gamma(1+(p+1) \alpha)}\right)^{\frac{1}{p}}\left(\frac{\Gamma(1+\alpha)}{\Gamma(1+2 \alpha)}\right)^{\frac{p-1}{p}}\left[\left|f^{(\alpha)}\left(x_{i}\right)\right|^{\frac{p}{p-1}}+\left|f^{(\alpha)}\left(x_{i+1}\right)\right|^{\frac{p}{p-1}}\right]^{\frac{p-1}{p}} .
\end{aligned}
$$

Hence in the trapezoidal case

$$
\begin{aligned}
& \left|T_{\alpha}\left(f, I_{n}\right)-{ }_{a} I_{b}^{(\alpha)} f(x)\right|=\left|\sum_{i=0}^{n-1}\left\{\frac{f\left(x_{i}\right)+f\left(x_{i+1}\right)}{2^{\alpha} \Gamma(1+\alpha)} l_{i}^{\alpha}-\frac{1}{\Gamma(1+\alpha)} \int_{x_{i}}^{x_{i+1}} f(s)(d s)^{\alpha}\right\}\right| \\
& \leq \sum_{i=0}^{n-1}\left|\frac{f\left(x_{i}\right)+f\left(x_{i+1}\right)}{2^{\alpha} \Gamma(1+\alpha)} l_{i}^{\alpha}-\frac{1}{\Gamma(1+\alpha)} \int_{x_{i}}^{x_{i+1}} f(s)(d s)^{\alpha}\right| \\
& \leq \sum_{i=0}^{n-1} \frac{l_{i}^{2 \alpha}}{2^{\alpha} \Gamma(1+\alpha)}\left(\frac{\Gamma(1+p \alpha)}{\Gamma(1+(p+1) \alpha)}\right)^{\frac{1}{p}}\left(\frac{\Gamma(1+\alpha)}{\Gamma(1+2 \alpha)}\right)^{\frac{p-1}{p}}\left[\left|f^{(\alpha)}\left(x_{i}\right)\right|^{\frac{p}{p-1}}+\left|f^{(\alpha)}\left(x_{i+1}\right)\right|^{\frac{p}{p-1}}\right]^{\frac{p-1}{p}} \\
& \leq \sum_{i=0}^{n-1} \frac{l_{i}^{2 \alpha}}{2^{\alpha} \Gamma(1+\alpha)}\left(\frac{\Gamma(1+p \alpha)}{\Gamma(1+(p+1) \alpha)}\right)^{\frac{1}{p}}\left(\frac{\Gamma(1+\alpha)}{\Gamma(1+2 \alpha)}\right)^{\frac{p-1}{p}} \max \left\{\left|f^{(\alpha)}\left(x_{i}\right)\right|,\left|f^{(\alpha)}\left(x_{i+1}\right)\right|\right\} \\
& \leq \frac{1}{2^{\alpha} \Gamma(1+\alpha)}\left(\frac{\Gamma(1+p \alpha)}{\Gamma(1+(p+1) \alpha)}\right)^{\frac{1}{p}}\left(\frac{\Gamma(1+\alpha)}{\Gamma(1+2 \alpha)}\right)^{\frac{p-1}{p}} \max \left\{\left|f^{(\alpha)}\left(x_{i}\right)\right|,\left|f^{(\alpha)}\left(x_{i+1}\right)\right|\right\} \sum_{i=0}^{n-1} l_{i}^{2 \alpha} .
\end{aligned}
$$

This completes the proof.

Proposition 24. Let $f$ be defined as in Corollary 18. Then we have

$$
\begin{aligned}
\left|E_{\alpha}\left(f, I_{n}\right)\right| & \leq \frac{1}{2^{2 \alpha}} \sum_{i=0}^{n-1}\left(\frac{\Gamma(1+\alpha)}{\Gamma(1+2 \alpha)}\left|f^{(\alpha)}\left(x_{i}\right)\right|+\frac{\Gamma(1+2 \alpha)}{\Gamma(1+3 \alpha)}\left|f^{(\alpha)}\left(x_{i+1}\right)\right|\right) l_{i}^{2 \alpha}\|g\|_{\left[x_{i}, x_{i+1}\right], \infty} \\
& \leq \frac{\max \left\{\frac{\Gamma(1+\alpha)}{\Gamma(1+2 \alpha)}\left|f^{(\alpha)}(a)\right|+\frac{\Gamma(1+2 \alpha)}{\Gamma(1+3 \alpha)}\left|f^{(\alpha)}(b)\right|\right\}}{2^{2 \alpha}} \sum_{i=0}^{n-1} l_{i}^{2 \alpha}\|g\|_{\left[x_{i}, x_{i+1}\right], \infty} .
\end{aligned}
$$

Proof. Apply Corollary 18 on $\left[x_{i}, x_{i+1}\right](i=0,1, \ldots, n-1)$ to get

$$
\begin{aligned}
& \left|f\left(x_{i}\right) \frac{1}{\Gamma(1+\alpha)} \int_{x_{i}}^{\frac{x_{i}+x_{i+1}}{2}} g(s)(d s)^{\alpha}+f\left(x_{i+1}\right) \frac{1}{\Gamma(1+\alpha)} \int_{\frac{x_{i}+x_{i+1}}{2}}^{x_{i+1}} g(s)(d s)^{\alpha}-\frac{1}{\Gamma(1+\alpha)} \int_{x_{i}}^{x_{i+1}} f(s)(d s)^{\alpha}\right| \\
& \leq\left(\frac{\Gamma(1+\alpha)}{\Gamma(1+2 \alpha)}\left|f^{(\alpha)}(a)\right|+\frac{\Gamma(1+2 \alpha)}{\Gamma(1+3 \alpha)}\left|f^{(\alpha)}(b)\right|\right) \frac{l_{i}^{2 \alpha}\|g\|_{\left[x_{i}, x_{i+1}\right], \infty}}{2^{2 \alpha}}
\end{aligned}
$$

Using (20), the generalized triangle and the generalized convexity of $\left|f^{(\alpha)}\right|$, we have the inequality (19). This completes the proof.

Remark 6. If we choose $g(t)=1^{\alpha}$ on $[a, b]$, then we the inequality (19) reduces to (17).

Proposition 25. Let $p>1$ and $f$ be defined as in Theorem 16. Then we have

$$
\begin{aligned}
\left|E_{\alpha}\left(f, I_{n}\right)\right| \leq & \frac{1}{2^{\alpha} \Gamma(1+\alpha)}\left(\frac{\Gamma(1+p \alpha)}{\Gamma(1+(p+1) \alpha)}\right)^{\frac{1}{p}}\left(\frac{\Gamma(1+\alpha)}{\Gamma(1+2 \alpha)}\right)^{\frac{p-1}{p}} \\
& \times \sum_{i=0}^{n-1}\left[\left|f^{(\alpha)}\left(x_{i}\right)\right|^{\frac{p}{p-1}}+\left|f^{(\alpha)}\left(x_{i+1}\right)\right|^{\frac{p}{p-1}}\right]^{\frac{p-1}{p}} l_{i}^{2 \alpha}\|g\|_{\left[x_{i}, x_{i+1}\right], \infty} \\
\leq & \frac{1}{2^{\alpha} \Gamma(1+\alpha)}\left(\frac{\Gamma(1+p \alpha)}{\Gamma(1+(p+1) \alpha)}\right)^{\frac{1}{p}}\left(\frac{\Gamma(1+\alpha)}{\Gamma(1+2 \alpha)}\right)^{\frac{p-1}{p}}
\end{aligned}
$$




$$
\times \max \left\{\left|f^{(\alpha)}\left(x_{i}\right)\right|,\left|f^{(\alpha)}\left(x_{i+1}\right)\right|\right\} \sum_{i=0}^{n-1} l_{i}^{2 \alpha}\|g\|_{\left[x_{i}, x_{i+1}\right], \infty} .
$$

Proof. The proof uses Corollary 21 and similar to that of Proposition 24.

Remark 7. If we choose $g(t)=1^{\alpha}$ on $[a, b]$, then we the inequality (21) reduces to (18).

\section{Some Inequalities For Random Variables}

Throughout this section, let $0<a<b, r \geq 1$, and let $X$ be a continuous random variable, $g(x) \in C_{\alpha}[a, b]$ be probability density function of $X$ which is symmetric to $(a+b) / 2$ and the $r-$ moment

$$
E_{r}^{\alpha}(X)=\frac{1}{\Gamma(\alpha+1)} \int_{a}^{b} s^{r \alpha} g(s)(d s)^{\alpha}
$$

which is assumed to be finite.

\section{Theorem 26.}

$$
\left|E_{r}^{\alpha}(X)-\frac{a^{r}+b^{r}}{2^{\alpha}} a_{b}^{(\alpha)} g(s)\right| \leq\left(\frac{\Gamma(1+\alpha)}{\Gamma(1+2 \alpha)} a^{r-1}+\frac{\Gamma(1+2 \alpha)}{\Gamma(1+3 \alpha)} b^{r-1}\right) \frac{r(b-a)^{2 \alpha}\|g\|_{[a, b], \infty}}{2^{2 \alpha}} .
$$

Proof. Let $f(s)=s^{r}(s \in[a, b])$ in Corollary 18. Then we have the following identities

$$
\begin{aligned}
\frac{1}{\Gamma(\alpha+1)} \int_{a}^{b} f(s) g(s)(d s)^{\alpha} & =E_{r}^{\alpha}(X), \\
\frac{f(a)+f(b)}{2^{\alpha}} \frac{1}{\Gamma(\alpha+1)} \int_{a}^{b} g(s)(d s)^{\alpha} & =\frac{a^{r}+b^{r}}{2^{\alpha}}{ }_{a} I_{b}^{(\alpha)} g(s)
\end{aligned}
$$

and

$$
\left|f^{(\alpha)}(a)\right|=r a^{r-1} \text { and }\left|f^{(\alpha)}(b)\right|=r b^{r-1} .
$$

Using the above identities and the inequality (11), we have the inequality (22). This completes the proof.

Remark 8. If we choose $r=1$ in Theorem 26 , then we have the following inequality

$$
\left|E^{\alpha}(X)-\frac{a+b}{2^{\alpha}}{ }_{a} I_{b}^{(\alpha)} g(s)\right| \leq\left(\frac{\Gamma(1+\alpha)}{\Gamma(1+2 \alpha)}+\frac{\Gamma(1+2 \alpha)}{\Gamma(1+3 \alpha)}\right) \frac{(b-a)^{2 \alpha}\|g\|_{[a, b], \infty}}{2^{2 \alpha}}
$$

where $E(X)$ is the generalized expectation of the random variable $X$.

\section{Conclusion}

We have extend some estimates of the right hand side of a Hermite-Hadamard-Fejér type inequality for generalized convex functions whose derivatives absolute values are generalized convex via local fractional integrals. We have introduced some inequalities for random variables and applications to weighted trapezoided formula on local fractional integrals.

Acknowledgments: The authors wishes to express his profound gratitude to the reviewers for their useful comments on the manuscript.

Author Contributions: All authors contributed equally to the writing of this paper. All authors read and approved the final manuscript.

Conflicts of Interest: "The authors declare no conflict of interest."

\section{References}

[1] Hadamard, J., (1893). Etude sur les proprietes des fonctions entieres et en particulier d'une fonction consideree par Riemann. Journal de Mathématiques Pures et Appliquées, 58, 171-215. 
[2] Bullen, P. S. (1978). Error estimates for some elementary quadrature rules. Publikacije Elektrotehnickog fakulteta. Serija Matematika i fizika, (602/633), 97-103.

[3] Dragomir, S. S. \& Pearce, C. E. M., (2000). Selected Topics on Hermite-Hadamard Inequalities and Applications. RGMIA Monographs, Victoria University.

[4] Mitrinovic, D. S., Pecaric, J., \& Fink, A. M. (2013). Classical and new inequalities in analysis (Vol. 61). Springer Science \& Business Media.

[5] Dragomir, S. S. \& Agarwal, R.P., (1998). Two inequalities for differentiable mappings and applications to special means of real numbers and to trapezoidal formula. Applied Mathematics Letters, 11(5), 91-95.

[6] El Farissi, A., Latreuch, Z. \& Belaidi, B. (2009). Hadamard-Type inequalities for twice diffrentiable functions. RGMIA Research Report collection, 1 12, art. 6.

[7] El Farissi, A. (2010). Simple proof and refinement of Hermite-Hadamard inequality. Journal of Mathematical Inequalities,4(3), 365-369.

[8] Gao, X. (2010). A note on the Hermite-Hadamard inequality. Journal of Mathematical Inequalities, 4(4), 587-591.

[9] Kirmaci, U. S., \& Ozdemir, M. E., (2004). On some inequalities for differentiable mappings and applications to special means of real numbers and to midpoint formula. Applied Mathematics and Computation, 153, 361-368.

[10] Kirmaci, U. S. \& Dikici, R., (2013). On some Hermite-Hadamard type inequalities for twice differentiable mappings and applications. Tamkang Journal of Mathematics ,1, 41-51.

[11] Kirmaci, U. S., (2004). Inequalities for differentiable mappings and applications to special means of real numbers and to midpoint formula. Applied Mathematics and Computation, 147, 137-146.

[12] Sarikaya, M. Z. \& Yaldiz, H., (2013). On the Hadamard's type inequalities for L-Lipschitzian mapping. Konuralp Journal of Mathematics,1(2), 33-40.

[13] Yang, X. J., (2012). Advanced Local Fractional Calculus and Its Applications. World Science Publisher, New York.

[14] Yang, Y. J., Baleanu, D., \& Yang, X. J. (2013). Analysis of fractal wave equations by local fractional Fourier series method. Advances in Mathematical Physics, Article ID 632309.

[15] Mo, H. Sui, X. \& Yu, D., (2014). Generalized convex functions on fractal sets and two related inequalities. Abstract and Applied Analysis, Article ID 636751, 7 pages.

[16] Sarikaya, M. Z., \& Budak, H. (2017). Some integral inequalities for local fractional integrals. International Journal of Analysis and Applications, 14(1), 9-19.

[17] Tseng, K. L., (2011). Some inequalities for differentiable mappings and applications to Fejér inequality and weighted trapezoidal formula. Taiwanese Journal of Mathematics ,14(4), 1737-1747.

[18] Sarikaya, M., \& Budak, H. (2017). Generalized Ostrowski type inequalities for local fractional integrals. Proceedings of the American Mathematical Society, 145(4), 1527-1538.

[19] Sarikaya, M. Z., Erden, S., \& Budak, H. (2016). Some generalized Ostrowski type inequalities involving local fractional integrals and applications. Advances in Inequalities and Applications, 2016, Article-63.

[20] Sarikaya, M. Z. \& Budak, H., (2015). On generalized Hermite-Hadamard inequality for generalized convex function. RGMIA Research Report Collection,18, Article 64, 15 pp.

[21] Sarikaya, M. Z., Budak, H., \& Erden, S. (2019). On new inequalities of SimpsonŠs type for generalized convex functions. Korean Journal of Mathematics, 27(2), 277-293. 